\title{
Angiogenic and FLT3 Receptors Expression in Acute Lymphoblastic Leukemia in Pediatric Age Group
}

\author{
Mona Hilmy Alrayes ${ }^{1 *}$, Reham Hammad ${ }^{*}{ }^{*}$, Mohamed Abd Alazim Hussein EL Baddiny ${ }^{2}$, \\ Maha Saleh Madbouly Ibrahim², Mahmoud Hammad ${ }^{3}$ \\ ${ }^{1}$ Clinical Pathology Department, Faculty of Medicine (for Girls), Al-Azhar University, Cairo, Egypt \\ ${ }^{2}$ Clinical Pathology Department, National Cancer Institute, Cairo University, Cairo, Egypt \\ ${ }^{3}$ Department of Pediatric Oncology, National Cancer Institute, Cairo University and Children's Cancer Hospital Egypt, Cairo, Egypt \\ Email: *mona_hilmy@yahoo.com, *dr_rema123@yahoo.com
}

How to cite this paper: Alrayes, M.H., Hammad, R., EL Baddiny, M.A.A.H., Ibrahim, M.S.M. and Hammad, M. (2019) Angiogenic and FLT3 Receptors Expression in Acute Lymphoblastic Leukemia in Pediatric Age Group. Journal of Cancer Therapy, 10, 442-457.

https://doi.org/10.4236/jct.2019.106037

Received: May 30, 2019

Accepted: June21, 2019

Published: June 24, 2019

Copyright $\odot 2019$ by author(s) and Scientific Research Publishing Inc. This work is licensed under the Creative Commons Attribution International License (CC BY 4.0).

http://creativecommons.org/licenses/by/4.0/

\begin{abstract}
Angiogenesis has an important role in pathophysiology of cancer. FMS-like tyrosine kinase 3 (FLT3) is implicated in hematopoietic malignancies. Their role in childhood acute lymphoblastic leukemia (ALL) pathogenesis needs more enlightenment. Expression of vascular endothelial growth factor receptor-1 and -2 (VEGFR-1 and -2), as well as FLT3 were assessed by flow cytometry in bone marrow (BM) blasts of 55 newly diagnosed children with ALL. Patients included B cell ALL (B-ALL) group $(n=41)$ and T cell ALL (T-ALL) group $(n=14)$. Comparison between groups revealed a significant increase in blasts percent (\%) expressing FLT3 and FLT3 intensity detected in B-ALL group ( $\mathrm{p}=0.004$ and $\mathrm{p}=0.02$, respectively). In B-ALL patients, a significant positive correlation was seen between blasts \% expressing FLT3 and blasts percentage infiltrating BM $(\mathrm{r}=0.405 ; \mathrm{p}=0.009)$, also positive correlation was seen between $\%$ of blasts expressing VEGFR-1 and VEGFR-2 $(\mathrm{r}=0.704 ; \mathrm{p}<0.001)$. In T-ALL group, blast \% expressing FLT3 revealed significant positive correlations with blast \% expressing VEGFR-1, and those expressing VEGFR-2 ( $\mathrm{r}=$ $0.627 ; p=0.016$, and $r=0.654 ; p=0.011$, respectively). In addition, significant correlation was seen in blasts \% expressing all; FLT3, VEGFR-1 and -2, with blasts \% expressing stem cell marker CD34 $(r=0.826 ; p=0.001, r=0.596 ; p$ $=0.041$, and $r=0.798 ; p=0.002$, respectively). Conclusion: Expression of VEGFR-1, VEGFR-2 and FLT3 were demonstrated and linked on leukemic blasts of ALL which highlights their role in pathogenesis. FLT3 expression plays a role in facilitating blasts proliferation in BM in B-ALL. FLT3, VEGFR-1 and -2 could be used in future profiling of $\mathrm{CD} 34^{+}$leukemic stem cell pool in T-ALL.
\end{abstract}




\section{Keywords}

Childhood Leukemia, Angiogenesis, Vascular Endothelial Growth Factor Receptors, FLT3, Acute Lymphoblastic Leukemia

\section{Introduction}

Childhood acute lymphoblastic leukemia (ALL) comprises about $30 \%$ of malignancies in childhood [1], and constitutes $80 \%$ of childhood leukemias [2]. In Egypt, the annual incidence of childhood ALL is about 4 cases per 100,000 children and accounts for approximately $20 \%$ of pediatric malignancies [3].

Many biomarkers are widely used for the diagnosis, as well as prediction of survival in cancer patients. The perturbation in expression of mediators and signaling pathways of angiogenesis have been described in the pathogenesis of hematological malignancies including acute and chronic leukemias, and multiple myeloma [4] [5]. The vascular endothelial growth factor (VEGF) family comprised of ligands and receptors those are key regulators of angiogenesis processes. The vascular endothelial growth factor receptors (VEGFRs), are members of the receptor tyrosine kinase (RTKs) and include three types; VEGFR-1-3. Seven VEGF ligand variants have been identified; VEGF-A-E and placental growth factor (PIGF) [6]. The VEGFR-1 (Flt-1) is located at chromosome 13q12 and VEGFR-2 (KDR/Flk-1) is located at chromosome 4q11 and both are expressed on vascular endothelial cells, whereas VEGFR-3 is expressed on lymphatic endothelial cells [7] [8].

FMS-like tyrosine kinase 3 (FLT3) represents another attractive RTK with its overexpression on the majority of hematopoietic progenitor cells, particularly in $\mathrm{CD}^{+}{ }^{+}$hematopoietic stem cells and leukemic cells. FLT3-ITD activating mutation is detected in $10 \%-15 \%$ of pediatric acute myeloid leukemia (AML) [9] [10]. FLT3 is aberrantly expressed in primary leukemia samples of almost all AML and in most of chronic myeloid leukemia (CML) blastic crisis [11].

Studies have related the expression of VEGF and its receptors, as well as FLT3 with prognosis and patients' survival in acute leukemia [12] [13] [14]. However, their role in pediatric ALL remains a point of debate. Herein, we aimed to study the expression pattern of VEGFR-1, VEGFR-2 and FLT3 receptors on leukemic blasts by flow cytometry in childhood ALL and to correlate it with other disease features.

\section{Patients and Methods}

This study was carried out on 55 children with ALL. The study was done in the department of pediatric oncology, National Cancer Institute (NCI); Cairo University in a one-year time period. All patients were newly diagnosed children with ALL below 18 years of age and selected before starting their first dose of chemotherapy. Previously treated and relapsing patients were excluded from the 
study. The study was approved by ethical committees of Faculty of Medicine; Al-Azhar University and National Cancer Institute; Cairo University.

Patients were divided into 2 groups: group (1) included 41 patients with B cell ALL (B-ALL) with male:female ratio $=1.2: 1$, and mean age of $5.97 \pm 4.84$. While group (2) included 14 patients with T cell ALL (T-ALL) with male:female ratio = $1: 1$ and mean age of $9.94 \pm 5.29$. Data collected included: a full history, clinical examination and initial laboratory workup in the form of complete blood picture (Sysmex KX21, Hematology analyzer, Kobe, Japan); examination of differential blood film for assessment of blast percentage (\%); bone marrow examination for morphology and assessment of blast \%, cytogenetic analysis and immunophenotyping of blast cells using standard leukemia panel including; CD34, HLA-DR, CD13, CD33, CD14, CD19, CD20, CD10, CD22, CD79a, cytoplasmic $\mu$ chain, CD2, cytoplasmic CD3, CD5, CD7, CD4 and CD8. Also, cytoplasmic myeloperoxidase (MPO) was tested for exclusion of myeloid nature association. Cells were considered positive, when the marker is expressed in $\geq 20 \%$ of cells except for CD34 where marker expression by $\geq 10 \%$ of the cells is sufficient to confer positivity.

Diagnosis was based on the presence of at least $20 \%$ blasts in the bone marrow (BM) film according to the WHO 2016 criteria, together with MPO negative immunophenotype results consistent with ALL. Remission status was evaluated at day 14 and day 42 from start of induction therapy. Complete remission was achieved when the BM is normocellular, with normal differential count and $<5 \%$ blasts.

Immunophenotyping assay: Flow cytometry assay was performed in NCI, using Coulter, Epics, XL Hialeh.

Immunophenotyping was performed on $2 \mathrm{ml}$ of $\mathrm{BM}$ sample transferred to EDTA tube before the administration of any treatment. The samples were analyzed within 24 hours of collection.

Assessment of FLT3, VEGFR-1 and VEGFR-2 was done after adjusting the sample count for acquisition, then 50 ug of adjusted sample was added to 4 tubes. Tube (1) was used for surface PE conjugated isotype control for detection of non-specific binding of surface FLT3. Tube (2) was used for detection of intracytoplasmic FITC and PE conjugated isotype controls for assessment of non-specific binding of intracytoplasmic VEGFR-1 and VEGFR-2 after following intracytoplasmic staining procedure. Tube (3) was used for assessment of PE conjugated anti-human FLT3 (Immunotech, Beckman Coulter company, Marseille, France, Catalog number: PNIM2234U. Clone: SF1340). Tube (4) was used for assessment of intracytoplasmic FITC conjugated anti-human VEGFR-1 (R\&D system Inc. Minneapolis, Minnesota, Catalog number: FAB321P. Lot number: LFR09) and PE conjugated anti-human VEGFR-2 (R\&D system Inc. Minneapolis, Minnesota, Catalog number: FAB357P. Lot number: LWS06).

Gating strategy: By using forward and side scatter (FS/SS), initial gating was performed on blasts area in the dot plot graph, then within the blast population, the subset of cells expressing FLT3, VEGFR-1 and VEGFR-2 were determined and their percentage was evaluated on quadrant histogram. Acquisition was set 
to 50,000 cells. Determining positive cut off was done using isotype control. In tube (3), FLT3 was determined on X axis, in tube (4) cytoplasmic VEGFR-1 was detected on $\mathrm{X}$ axis and cytoplasmic VEGFR-2 was determined on Y axis, area of co-expression was determined on upper right quadrant. Data were also expressed as mean fluorescence intensity (MFI) of each of FLT3, VEGFR-1 and VEGFR-2 using single histogram (Figure 1).
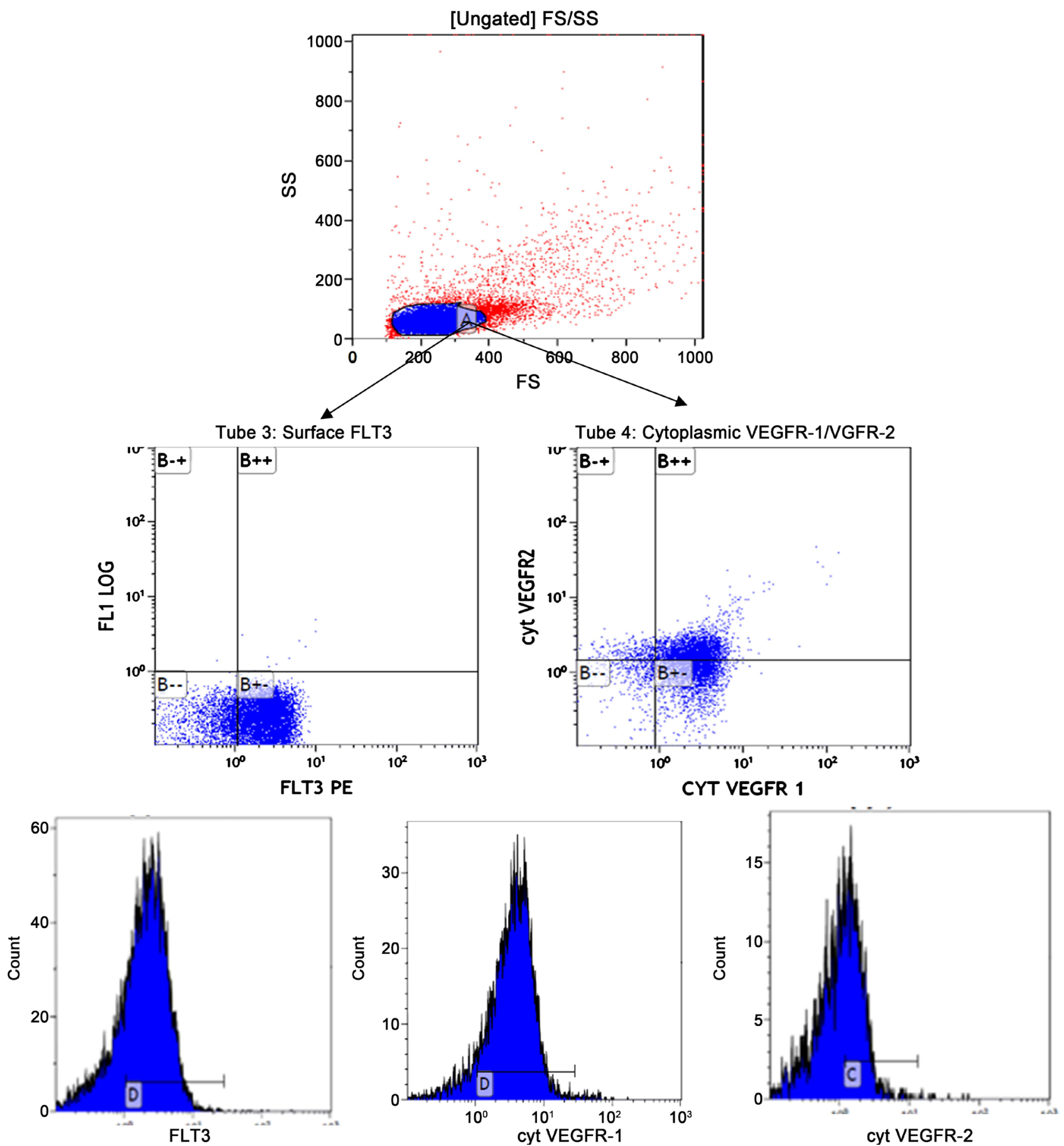

Figure 1. Gating strategy for assessing percent of blasts expressing surface FLT3, cytoplasmic VEGFR-1 and cytoplasmic VEGFR-2 and single histogram for assessing the mean fluorescence intensity (MFI) for each marker. 


\section{Statistical Methods}

All statistical calculations were done using computer programs SPSS (Statistical Package for the Social Science; SPSS Inc., Chicago, IL, USA) version 15 for Microsoft Windows. Data were statistically described in terms of mean \pm standard deviation $( \pm S D)$, median and range, or frequencies (number of cases) and percentages when appropriate. Comparison of the mean of the parameters between the study groups was performed using T-test. Comparison of numerical variables between the study groups was done using Mann Whitney $U$ test for independent samples. Relation between different numerical variables was tested using Pearson correlation. Correlation coefficient is referred to as (r). Probability (p-values) less than 0.05 was considered statistically significant.

\section{Results}

Clinical and laboratory data of patients are summarized in Table 1 and Table 2. The total B-ALL patients evaluated for their initial studied parameters were forty-one patients, with $22(53.7 \%)$ males and 19 (46.3\%) female patients (male:female = 1.2:1). The age ranged from 4 months to 17 years (mean $\pm \mathrm{SD}=5.97 \pm 4.84$ ). We managed to follow only 36 patients. Out of 36 patients, four patients died during induction before reaching evaluation. At day 14, the initial response to chemotherapy was assessed and 30/32 (93.8\%) patients were in remission, while 2/32 (6.2\%) patients were not in remission. Eight patients died between day 14 and day 42 . The remaining 24 patients were assessed at day 42 (end of induction phase), and all of them had achieved complete remission (CR). The duration between the maximal response to chemotherapy till progression was called time to disease progression (TDP), of those 24 patients who were in CR at day 42 only $1 / 24$ (4.2\%) patient relapsed before death. By the end of follow up period, 23/36 (63.9\%) patients were alive.

The total T-ALL patients evaluated for their initial studied parameters were fourteen patients, with 7 (50\%) males (male: female ratio 1:1). The age ranged from 1.5 years to 16 years (mean $\pm \mathrm{SD}=9.94 \pm 5.29$ ). Only 11 patients were followed and assessed for their induction chemotherapy response. At day 14 evaluation, 10/11 (93\%) patients were in remission, while only one patient was not in remission. At day 42 evaluation, all patients achieved complete remission. After entering CR only 2/11(18.2) patient relapsed before the end of the study and died after relapse. By the end of the study, nine (81.8\%) patients were alive.

Comparison of studied parameters between the two groups is detailed in Table 3. There was a significant increase of $\%$ of blasts expressing FLT3 and FLT3 (MFI) detected in B-ALL when compared to T-ALL.

In B-ALL group, splenomegaly, hepatomegaly, and lymphadenopathy were clinically detected in 27/36,30/36, and 27/41. respectively. Only patients presenting with splenomegaly showed a statistically significant decrease in percentages 
Table 1. Clinical data of childhood ALL patients.

\begin{tabular}{cccc}
\hline Clinical data (Total 55 patients) & & Frequency & Percentage (\%) \\
\hline Sex & Male & 29 & $52.7 \%$ \\
Ratio 1.1:1 & Female & 26 & $47.3 \%$ \\
General symptoms (n = 55) & Present & 42 & $76.3 \%$ \\
(fever, pallor, ecchymosis, bleeding) & Absent & 13 & $23.7 \%$ \\
Lymphadenopathy (n = 55) & Present & 36 & $65.5 \%$ \\
Hepatomegaly & Absent & 19 & $34.5 \%$ \\
Splenomegaly & Present & 38 & $77.6 \%$ \\
(US was performed for 49 patients) & Absent & 11 & $22.4 \%$ \\
Mediastinal mass & Present & 38 & $77.6 \%$ \\
(US was performed for 49 patients) & Absent & 11 & $22.4 \%$ \\
CNS infiltration & Present & 3 & $10 \%$ \\
Chest $\times$ ray was performed for 30 patients) & Absent & 27 & $90 \%$ \\
(CSF examination was done for 50 patients) & Present & 4 & $8 \%$ \\
WHO classification (n $=55)$ & Absent & 46 & $92 \%$ \\
& B-ALL & 41 & $74.5 \%$ \\
& Pro-B-ALL & 2 & $4.8 \%$ \\
& Common B-ALL & 7 & $17.1 \%$ \\
& Pre-B-ALL & 32 & $78.1 \%$ \\
& T-ALL & 14 & $25.5 \%$ \\
\hline
\end{tabular}

US, ultrasound; CSF, cerebrospinal fluid; CNS, central nervous system; WHO, world health organization.

Table 2. Age and laboratory data of the total cohort.

\begin{tabular}{cccccc}
\hline Parameters & Number & Frequency & Mean \pm SD & Range & Median \\
\hline Age & 55 & $100 \%$ & $2.09 \pm 0.67$ & $0.33-17$ & \\
$\leq 1$ year & 1 & $1.8 \%$ & $0.7 \pm 0.42$ & $0.33-1$ & 0.7 \\
$>1-\leq 10$ year & 39 & $70.9 \%$ & $4.28 \pm 2.21$ & $1-10$ & 4 \\
$>10$ year & 15 & $27.3 \%$ & $14.67 \pm 1.72$ & $11-17$ & 15 \\
TLC & 55 & $100 \%$ & $76.22 \pm 140.19$ & $0.8-579$ & \\
$<10\left(\times 10^{9} / \mathrm{L}\right)$ & 17 & $30.9 \%$ & $3.64 \pm 2.80$ & $0.8-10$ & 2.90 \\
$\geq 10-\leq 50\left(\times 10^{9} / \mathrm{L}\right)$ & 21 & $38.2 \%$ & $18.68 \pm 9.08$ & $10.35-47$ & 16.60 \\
$>50\left(\times 10^{9} / \mathrm{L}\right)$ & 17 & $30.9 \%$ & $219.86 \pm 185.37$ & $54-579$ & 112.00 \\
Hb & 55 & $100 \%$ & $8.39 \pm 2.25$ & $3.4-14$ & \\
$<8$ gm/dl & 26 & $49.1 \%$ & $6.59 \pm 1.15$ & $3.4-8$ & 6.8 \\
$\geq 8$ gm/dl & 27 & $50.9 \%$ & $10.15 \pm 1.54$ & $8.1-14$ & 9.7 \\
PLT & 55 & $100 \%$ & $72.56 \pm 84.59$ & $6-369$ & \\
$<150$ & 50 & $90.9 \%$ & $49.51 \pm 38.40$ & $6-134$ & 41 \\
$\geq 150$ & 5 & $9.1 \%$ & $303 \pm 74.24$ & $181-369$ & 329 \\
BM blasts $(\%)$ & 55 & 100 & $84.33 \pm 13.11$ & $25-98$ & 85 \\
PB blasts $(\%)$ & 42 & $76.4 \%$ & $39.53 \pm 36.79$ & $0-97$ & 35 \\
\hline
\end{tabular}

TLC, total leukocytic count; Hb, Hemoglobin, PLT, platelet; BM, bone marrow; PB, peripheral blood. 
Table 3. B-ALL and T-ALL groups as regards the expression of markers (VEGFR-1/-2 and FLT3).

\begin{tabular}{cccccc}
\hline \multirow{2}{*}{ Parameters } & \multicolumn{2}{c}{ B cell ALL $(\mathrm{n}=41)$} & \multicolumn{2}{c}{ T cell ALL $(\mathbf{n}=14)$} & \\
\cline { 2 - 6 } & Mean \pm SD & Range & Mean \pm SD & Range & \\
\hline \% of blasts expressing FLT3 & $45.75 \pm 23.85$ & $1.44-84$ & $23.62 \pm 22.82$ & $2.70-75.5$ & $\mathbf{0 . 0 0 4}$ \\
FLT3 (MFI) & $2.17 \pm 0.71$ & $1.13-4.19$ & $1.67 \pm 0.46$ & $1.07-3.06$ & 0.02 \\
\% of blasts expressing VEGFR-1 & $76.35 \pm 12.98$ & $40.6-99.5$ & $69.73 \pm 20.59$ & $31.1-97.10$ & 0.17 \\
VEGFR-1 (MFI) & $4.33 \pm 2.65$ & $1.86-15$ & $3.62 \pm 1.97$ & $1.65-9.85$ & 0.36 \\
\% of blasts expressing VEGFR -2 & $40.64 \pm 23.52$ & $3.46-98.7$ & $29.72 \pm 26.53$ & $3.37-88.30$ & 0.15 \\
VEGFR -2 (MFI) & $2.39 \pm 2.11$ & $1.03-14$ & $1.92 \pm 0.59$ & $1.30-3.430$ & 0.42 \\
\hline
\end{tabular}

FLT3, FMS-like tyrosine kinase 3; MFI, mean fluorescence intensity; VEGFR, vascular endothelial growth factor receptors.

of blasts expressing VEGFR-1 $(\mathrm{p}=0.014)$, and VEGFR-2 $(\mathrm{p}=0.009)$ as well as the MFI of VEGFR-1 ( $p=0.006)$, and VEGFR-2 $(\mathrm{p}=0.006)$ compared to patients without splenomegaly. While no significant difference detected in both groups in \% of blasts expressing FLT3 ( $\mathrm{p}=0.333$ ) or FLT3 MFI (0.257). Also, no statistically significant difference was observed for the studied markers in the B-ALL group between patients with hepatomegaly and lymphadenopathy and those without.

In B-ALL patients, a significant positive correlation was seen between $\%$ of blasts expressing FLT3 and \% of blasts infiltrating BM $(r=0.405 ; p=0.009)$ (Figure 2(A)). Also, blasts percent expressing VEGFR-2 was significantly correlated with $\mathrm{CD} 10 \%(\mathrm{r}=0.379 ; \mathrm{p}=0.015)$, as well as between \% of blasts expressing VEGFR-1 and VEGFR-2 $(\mathrm{r}=0.704$; $\mathrm{p}<0.001)$ (Figure 3(A)), while no correlation was seen with any of the studied markers with the $\%$ of blasts expressing stem cell marker CD34.

In the T-ALL group, blast percent expressing FLT3 revealed significant positive correlations with the blast percent expressing VEGFR-1\%, and those expressing VEGFR-2\% $(\mathrm{r}=0.627 ; \mathrm{p}=0.016$, and $\mathrm{r}=0.654 ; \mathrm{p}=0.011$, respectively $)$ (Figure 2(B) and Figure 2(C)). Positive correlation was observed between FLT3\% and the stem cell marker CD34 $(r=0.826 ; p=0.001)$ (Supplementary Figure $\mathrm{S} 1(\mathrm{~A})$, online version) There was significant positive correlation between blast percent expressing VEGFR-1 and VEGFR-2 $(r=0.762 ; \mathrm{p}=0.002)$ (Figure $3(B)$ ) and also significance was seen with \% of blasts expressing stem cell marker CD34 and each of \% of blasts expressing VEGFR-1 and \% of blasts expressing VEGFR-2 $(\mathrm{r}=0.596 ; \mathrm{p}=0.041$, and $\mathrm{r}=0.798 ; \mathrm{p}=0.002$, respectively) (supplementary Figure S1(B) and Figure S1(C), respectively, online version).

\section{Discussion}

Childhood acute lymphoblastic leukemia is the most common hematological malignancy, accounting approximately for $80 \%$ of all childhood leukemias. There has been a gradual increase in the incidence of ALL in the past 25 years, 
(A)

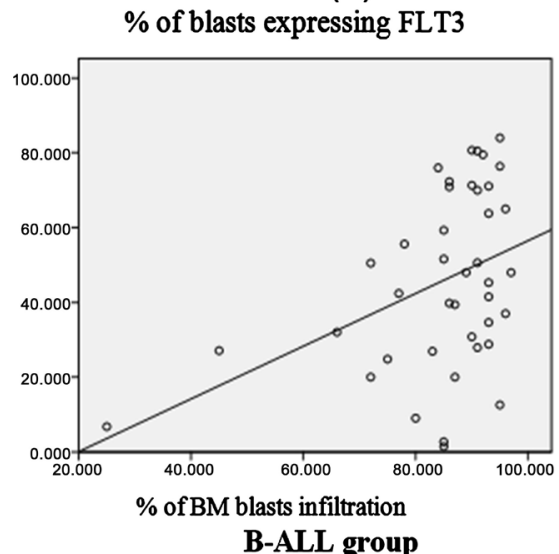

(B)

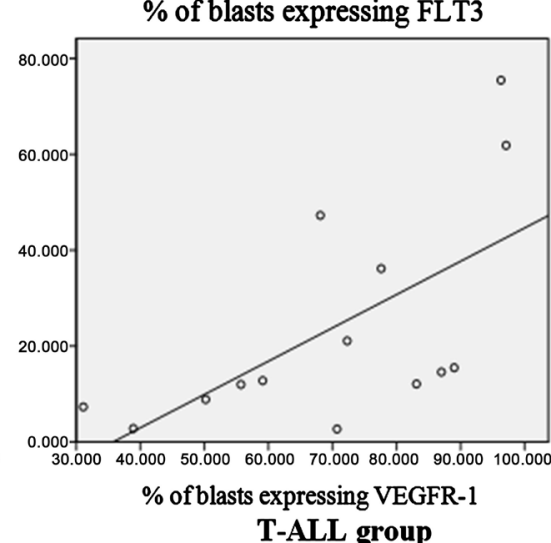

(C)

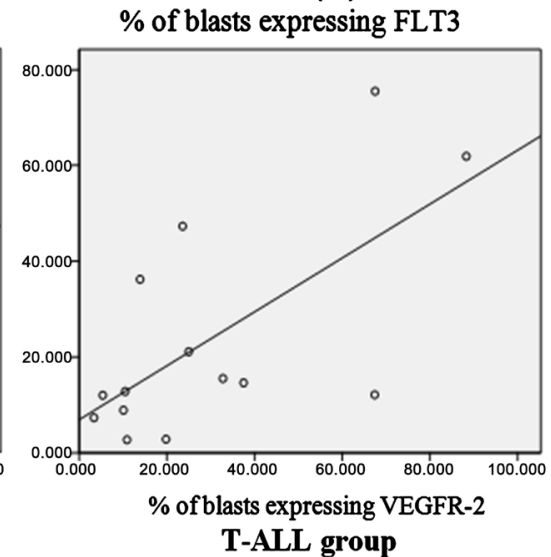

Figure 2. Correlation between \% of blasts expressing FLT3 and \% of BM blasts in B-All cases, \% of blasts expressing VEGFR-1 in T-ALL, \% of blasts expressing VEGFR-2 in T-ALL. (A) Positive correlation between $\%$ of blasts expressing FLT3 and \% of BM blasts in B-All cases $(r=0.405 ; p=0.009)$. (B) Positive correlation between $\%$ of blasts expressing FLT3 and \% of blasts expressing VEGFR-1 in T-ALL ( $r=0.0 .627 ; \mathrm{p}=0.016)$. (C) Positive correlation between \% of blasts expressing FLT3 and \% of blasts expressing VEGFR-2 in T-ALL $(\mathrm{r}=0.654 ; \mathrm{p}=0.011)$.

(A)

$\%$ of blasts expressing VEGFR-1

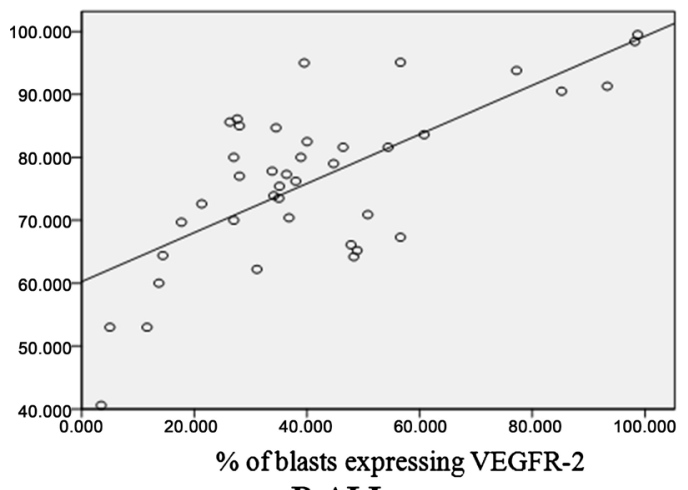

B-ALL group
(B)

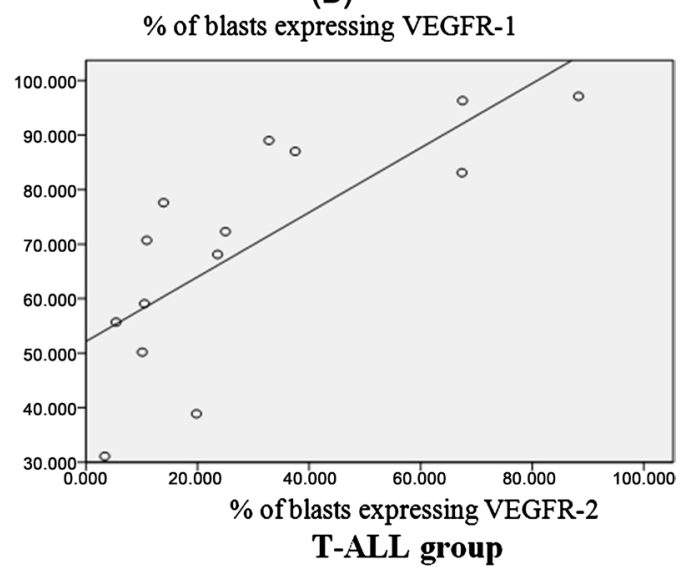

Figure 3. Correlation between \% of blasts expressing VEGFR-1 and \% of blasts expressing VEGFR-2 in B-ALL group and T-ALL group. (A) Positive correlation between \% of blasts expressing VEGFR-1 and \% of blasts expressing VEGFR-2 in B-ALL group $(r=$ 0.704; $\mathrm{p}<0.001$ ). (B) Positive correlation between $\%$ of blasts expressing VEGFR-1 and \% of blasts expressing VEGFR-2 in T-ALL group $(\mathrm{r}=0.762 ; \mathrm{p}=0.002)$.

however with newly modified treatment strategies high cure rates $>80 \%$ have been achieved [15]. Researches recognized the important role of angiogenesis in the pathogenesis of leukemias and solid tumors and as a potential treatment target [16]. Decreased survival and lower remission rates have been linked with the expression levels of RTK; VEGFR-1 and -2, in patients with different hematolymphoid tumors [17]. However, in pediatric ALL the expression of angiogenic molecules and its relation to disease features are still not fully assessed.

Another RTK, FMS-like tyrosine kinase 3 (FLT3), is predominantly expressed on hematopoietic progenitor cells. It has a significant role in both differentiation and proliferation of hematopoietic progenitor cells [18].

In the current work, we have evaluated the level of expression of VEGFR-1 
and VEGFR-2, as well as FLT3 receptors by flow cytometry on leukemic blasts of 55 pediatric patients with B- and T- cell ALL. The mean age of the total studied patients was $2.09 \pm 0.67$ years with a male: female ratio of 1.1:1. Similarly, others reported that the incidence of ALL among children aged 2 to 5 years is approximately fourfold greater than other age groups [19].

A significant increase was observed in \% of blasts expressing FLT3 and in FLT3 (MFI) in B-ALL cases compared to T-ALL ( $p=0.004$ and $p=0.02$, respectively). The observation was in line with a previous study, which tested the expression of FLT3 mRNA and its cell surface expression by flow cytometry and reported a higher frequency of FLT3 expression by flow cytometry on leukemic blasts in B-ALL (64\%) compared to T-ALL (30\%). While at the mRNA level, FLT3 was expressed in $80 \%-100 \%$ of leukemic cases in both phenotypes [20]. A similar conclusion was reached by other authors, who observed the higher expression of FLT3 in B-ALL compared to T-ALL using flow cytometry assay and immunoblotting technique in childhood B-ALL [21] and adulthood B-ALL [22], strengthening the clinical importance for FLT3 in pathogenesis of B-ALL

Even though it was not statistically significant, still we observed a higher mean \pm SD value for VEGFR-1 and VEGFR-2 in B-ALL $(76.35 \% \pm 12.98 \%$ and $40.64 \%$ $\pm 23.52 \%$, respectively) than in T-ALL $(69.73 \% \pm 20.59 \%$ and $29.72 \% \pm 26.72 \%$, respectively) $(\mathrm{p}=0.17)$. The same expression patterns were detected by El-Obeid et al., who reported that both VEGFR-1 and VEGFR-2 are concomitantly expressed in both ALL phenotype cell lines but primary in precursor B-ALL using flow cytometry and added that their expression is limited to the intra-cytoplasmic compartment which may suggest either internalization of the receptors or a block in trafficking of the receptor to the surface [13]. Also, another study reported that notably, pre-B ALL patients had significantly increased expression of VEGFR-1 compared with no expression in the nonmalignant group [5].

Extramedullary involvement occurs in $20 \%$ of ALL patients at presentation. Common sites include central nervous system and testicular involvement, as well as organomegaly and lymphadenopathy [23]. The number of patients with cerebrospinal fluid (CSF) infiltration in this study was detected only in four patients out of 50 cases with available CSF analysis, which is a small number to be statistically analyzed. Splenomegaly, hepatomegaly and lymphadenopathy were clinically detected in 38/49,38/49 and 36/55 cases, respectively and despite that organ involvement is not included in recent protocols as a risk feature, except for the central nervous system and testis, but some studies observed that it can still decrease remission and increase relapse rates [24].

In B-ALL cases, the expression pattern of FLT3 in this study did not show statistical significant difference between those who had extramedullary involvement and those without. Unlike others who reported the ability of the FLT3 to facilitate the migration of bone marrow blasts to the extramedullary sites through modulating FLT3 ligand and CXCL12/CXC4 axis [25].

In contrast, in the B-ALL cases with splenomegaly, a significant decrease in 
blasts percent expressing both VEGFR-1 and VEGFR-2 ( $\mathrm{p}=0.014$ and $\mathrm{p}=0.009$ respectively) with a decrease of their MFI too when compared with cases without splenomegaly. Contrary to our finding, Dias et al. study, observed the overexpression of VEGFRs in organomegaly and activation of VEGF/VEGFR-1-2 axis which induces matrix metalloproteinase (MMP) production that leads to the migration of blast cells to extramedullary sites [26]. Other authors reported that, VEGFR-1 signaling only was involved in precursor B cells mobility from bone marrow to spleen, whereas VEGFR-2 was more involved in cell differentiation [27]. A possible explanation for the difference in results was discussed by others, who had concluded that overexpression of VEGFR-1 in blast cells of ALL results in their migration to extramedullary sites, whereas blast cells not expressing VEGFR-1 tend to localize in bone marrow, and in the current study only BM localized blasts were examined [5] [28]. Another explanation could be attributed to the formation of soluble forms of VEGFRs (sVEGFR) by detachment of the extra cellular part of the receptor to the plasma as a negative feedback mechanism to decrease the level of expression of the whole receptor on the blasts and consequently their activity. sVEGFR were found to correlate to metastasis and central nervous system migration of blast cells in Tang et al. study [29]. Those soluble forms of receptors were not assessed in the present study.

Statistically, significant correlation was observed, between blast percent expressing FLT3 and percent of blasts infiltrating the bone marrow in B-ALL patients $(\mathrm{r}=0.405 ; \mathrm{p}=0.009)$ (Figure $2(\mathrm{~A})$ ). This finding was supported by other studies, which reported that human FLT3 signaling prevents stem cells and lymphoid progenitors from spontaneous apoptotic cell death at least through up-regulating MCL-1, which increases survival of those blasts [30] [31].

No correlation was seen between blast percent expressing FLT3 and blast percent expressing CD34 in B-ALL cases $(r=0.099, \mathrm{p}=0.552)$, this disagreed with Vora et al., who reported that CD34 expression was associated with FLT3 expression in B-ALL [21].

In the B-ALL group, a positive correlation was seen between $\%$ of blasts expressing VEGFR-1 and \% of blasts expressing VEGFR-2 ( $\mathrm{r}=0.704 ; \mathrm{p}<0.001)$. Similarly, positive correlation was found in the T-ALL group between $\%$ of blasts expressing VEGFR-1 and VEGFR-2 ( $\mathrm{r}=0.762$; $\mathrm{p}=0.002$ ) (Figure 3(A) and Figure 3(B)). This finding ties well with Scavelli et al., who proposed the crosstalk between angiogenesis and proliferation of lymphatic cells [32] and throws light on a the link between receptor tyrosine kinase (RTK)-dependent signaling pathways which was proven to be implicated in the pathogenesis of ALL of childhood and strengthen the role of their combined inhibition in controlling the biological behavior of leukemia as suggested by others [33].

In T-ALL group, blast percent expressing FLT3 revealed significant positive correlations with both VEGFR-1 and VEGFR-2, $(\mathrm{r}=0.627 ; \mathrm{p}=0.016$, and $\mathrm{r}=$ $0.654 ; \mathrm{p}=0.011$, respectively) (Figure 2(B) and Figure $2(\mathrm{C})$ ). Others found a positive correlation for the expression of FLT3 with somatic gene mutations in 
T-ALL. Giving FLT3 surface expression importance in prediction of molecular aberrations in T-ALL settings [34] [35]. Combining the observation of expression of VEGFR-1-2 with FLT3 expression may highlight more facts for the detection of molecular aberrations in T-ALL in the future. Interestingly, in this current study, FLT3 expression in the T-ALL was positively correlated with the stem cell marker CD34 $(r=0.826 ; p=0.001)$ (Supplementary Figure $\mathrm{S} 1(\mathrm{~A})$ ). Similarly, Drexler et al. observed a restricted FLT3 expression seen on human hematopoietic progenitor cells $\mathrm{CD} 34^{+}[20]$. Also, positive correlation was observed in T-ALL cases between \% of blasts expressing CD34 with \% of blasts expressing both VEGFR-1 and VEGFR-2, $(\mathrm{r}=0.569 ; \mathrm{p}=0.041)$ and $(\mathrm{r}=0.798 ; \mathrm{p}=$ 0.002), respectively Supplementary Figure S1(B) and Figure S1(C)). Previous observation may contribute to the emerging of new targeted therapies directed to leukemic stem cell (LSC). The LSC-hypothesis assumes that leukemias are organized hierarchically, with more mature cells programmed to undergo apoptosis after a limited number of cell divisions, and LSCs which have self-renewal and thus unlimited disease-propagating ability. In adult ALL, the phenotype of LSCs is supposed to reside within a CD $34^{+}$compartment. However, in childhood ALL, little is known about markers and target expression profiles in LSCs [36]. Finding markers associated with $\mathrm{CD}_{3} 4^{+}$compartment may help in profiling of LSCs to be used in future curative targeted therapies.

There was no significant correlation between any of the angiogenic markers and CD34 expressing blasts in B-ALL. Similarly, others concluded that, VEGFR-1 was expressed by more mature blasts committed to B lineage, which are mostly CD34 negative cells [37]. This ties well with our finding, that demonstrated a positive correlation between the blast \% expressing VEGFR-2 and those expressing CD10 ( $\mathrm{r}=0.379 ; \mathrm{p}=0.015)$ as CD10 is gained in more mature blasts.

The strength of this study, is related to its prospective design in newly diagnosed pediatric ALL cases, with few studies discussing our point in this age group. Another strength point was studying the expression of angiogenic markers (VEGFR1-2), in addition to FLT3 expression in leukemic cells, describing a link between angiogenesis and lymphatic cell proliferation in ALL. An apparent limitation in the current study is related to the small number of patients included in both groups, B-ALL and T-ALL, besides the assessment of biomarkers expressed were not studied in relation to the disease outcome and response to treatment. These limitations come from the financial restrictions that limit our ability to repeat the assessment of markers expression in a larger group of patients and at different treatment phases, but at least it points to the importance of studying the previously included biomarkers that could be associated with the pathogeneses of ALL and its survival outcome.

In conclusion: Expression of VEGFR-1, VEGFR-2 and FLT3 were demonstrated on leukemic blasts of ALL which highlights their role in pathogenesis. FLT3 expression plays a role in facilitating blasts proliferation in the bone marrow of childhood B-ALL. FLT3 positive correlations with both VEGFR-1 and VEGFR-2 
in T-ALL cases spotted a link between angiogenesis and the FLT3 proliferative ability in the pathogenesis of T-ALL. Usefulness of FLT3, VEGFR-1, and VEGFR-2 in future identification of $\mathrm{CD} 34^{+}$pool of blasts and profiling of leukemic stem cell (LSCs) in T-ALL. In addition, further prospective studies are needed to prove the prognostic significance of such biomarkers on the survival outcome in childhood ALL.

\section{Conflicts of Interest}

The authors declare no conflicts of interest regarding the publication of this paper.

\section{References}

[1] Faderl, S., O'Brien, S., Pui, C.H., Stock, W., Wetzler, M., Hoelzer, D. and Kantarjian, H.M. (2010) Adult Acute Lymphoblastic Leukemia: Concepts and Strategies. Cancer, 116, 1165-1176. https://doi.org/10.1002/cncr.24862

[2] Ezzat, S., Rashed, W.M., Salem, S., Dorak, M.T., El-Daly, M., Abdel-Hamid, M., Sidhom, I., El-Hadad, A. and Loffredo, C. (2016) Environmental, Maternal, and Reproductive Risk Factors for Childhood Acute Lymphoblastic Leukemia in Egypt: A Case-Control Study. BMC Cancer, 16, 662. https://doi.org/10.1186/s12885-016-2689-Z

[3] Adnan Awad, S., Kamel, M.M., Ayoub, M.A., Kamel, A.M., Elnoshokaty, E.H. and El Hifnawi, N. (2016) Immunophenotypic Characterization of Cytogenetic Subgroups in Egyptian Pediatric Patients with B-Cell Acute Lymphoblastic Leukemia. Clinical Lymphoma, Myeloma and Leukemia, 16, S19-S24.e1. https://doi.org/10.1016/j.clml.2016.02.032

[4] Leblebisatan, G., Antmen, B., Şaşmaz, I. and Kilinç, Y. (2012) Vascular Endothelial Growth Factor Levels in Childhood Acute Lymphoblastic and Myeloblastic Leukemia. Indian Journal of Hematology and Blood Transfusion, 28, 24-28. https://doi.org/10.1007/s12288-011-0102-2

[5] Diffner, E., Gauffin, F., Anagnostaki, L., Nordgren, A., Gustafsson, B., Sander, B., Gustafsson, B. and Persson, J.L. (2009) Expression of VEGF and VEGF Receptors in Childhood Precursor B-Cell Acute Lymphoblastic Leukemia Evaluated by Immunohistochemistry. Journal of Pediatric Hematology/Oncology, 31, 696-701. https://doi.org/10.1097/MPH.0b013e3181b258df

[6] Persson, A.B. and Buschmann, I.R. (2011) Vascular Growth in Health and Disease. Frontiers in Molecular Neuroscience, 4, 14. https://doi.org/10.3389/fnmol.2011.00014

[7] Murakami, M., Zheng, Y., Hirashima, M., Suda, T., Morita, Y., Ooehara, J., Ema, H., Fong, G.-H. and Shibuya, M. (2008) VEGFR1 Tyrosine Kinase Signaling Promotes Lymphangiogenesis as Well as Angiogenesis Indirectly via Macrophage Recruitment. Arteriosclerosis, Thrombosis, and Vascular Biology, 28, 658-664. https://doi.org/10.1161/ATVBAHA.107.150433

[8] Hiratsuka, S., Kataoka, Y., Nakao, K., Nakamura, K., Morikawa, S., Tanaka, S., Katsuki, M., Maru, Y. and Shibuya, M. (2005) Vascular Endothelial Growth Factor A (VEGF-A) Is Involved in Guidance of VEGF Receptor-Positive Cells to the Anterior Portion of Early Embryos. Molecular and Cellular Biology, 25, 355-363. https://doi.org/10.1128/MCB.25.1.355-363.2005

[9] Annesley, C.E. and Brown, P. (2014) The Biology and Targeting of FLT3 in Pedia- 
tric Leukemia. Frontiers in Oncology, 4, 263.

https://doi.org/10.3389/fonc.2014.00263

[10] Kondo, M., Horibe, K., Takahashi, Y., Matsumoto, K., Fukuda, M., Inaba, J., Kato, K., Kojima, S. and Matsuyama, T. (1999) Prognostic Value of Internal Tandem Duplication of the FLT3 Gene in Childhood Acute Myelogenous Leukemia. Medical and Pediatric Oncology, 33, 525-529. https://doi.org/10.1002/(SICI)1096-911X(199912)33:6<525::AID-MPO1>3.3.CO;2-\#

[11] Rosnet, O., Bühring, H.J., Marchetto, S., Rappold, I., Lavagna, C., Sainty, D., Arnoulet, C., Chabannon, C., Kanz, L., Hannum, C. and Birnbaum, D. (1996) Human FLT3/FLK2 Receptor Tyrosine Kinase Is Expressed at the Surface of Normal and Malignant Hematopoietic Cells. Leukemia, 10, 238-248.

[12] Karabacak, B.H., Erbey, F., Bayram, I., Yilmaz, S., Acipayam, C., Kilinç, Y. and Tanyeli, A. (2010) Fms-Like Tyrosine Kinase 3 Mutations in Childhood Acute Leukemias and Their Association with Prognosis. Asian Pacific Journal of Cancer Prevention, 11, 923-927.

[13] El-Obeid, A., Sunnuqrut, N., Hussain, A., Al-Hussein, K., Gutiérrez, M.I. and Bhatia, K. (2004) Immature B Cell Malignancies Synthesize VEGF, VEGFR-1 (Flt-1) and VEGFR-2 (KDR). Leukemia Research, 28, 133-137. https://doi.org/10.1016/S0145-2126(03)00188-7

[14] Aguayo, A., Estey, E., Kantarjian, H., Mansouri, T., Gidel, C., Keating, M., Giles, F., Estrov, Z., Barlogie, B. and Albitar, M. (1999) Cellular Vascular Endothelial Growth Factor Is a Predictor of Outcome in Patients with Acute Myeloid Leukemia. Blood, 94, 3717-3721.

[15] Siddaiahgari, S., Awaghad, M. and Latha, M. (2015) Clinical, Immunophenotype and Cytogenetic Profile of Acute Lymphoblastic Leukemia in Children at Tertiary Health Care Centre in India. Muller Journal of Medical Sciences and Research, 6, 112. https://doi.org/10.4103/0975-9727.160676

[16] Stachel, D., Albert, M., Meilbeck, R., Paulides, M. and Schmid, I. (2007) Expression of Angiogenic Factors in Childhood B-Cell Precursor Acute Lymphoblastic Leukemia. Oncology Reports, 17, 147-152. https://doi.org/10.3892/or.17.1.147

[17] Song, G., Li, Y. and Jiang, G. (2012) Role of VEGF/VEGFR in the Pathogenesis of Leukemias and as Treatment Targets (Review). Oncology Reports, 28, 1935-1944. https://doi.org/10.3892/or.2012.2045

[18] Carow, C.E., Levenstein, M., Kaufmann, S.H., Chen, J., Amin, S., Rockwell, P., Witte, L., Borowitz, M.J., Civin, C.I. and Small, D. (1996) Expression of the Hematopoietic Growth Factor Receptor FLT3 (STK-1/Flk2) in Human Leukemias. Blood, 87, 1089-1096.

[19] Pui, C.-H., Robison, L.L. and Look, A.T. (2008) Acute Lymphoblastic Leukaemia. The Lancet, 371, 1030-1043. https://doi.org/10.1016/S0140-6736(08)60457-2

[20] Drexler, H.G. (1996) Expression of FLT3 Receptor and Response to FLT3 Ligand by Leukemic Cells. Leukemia, 10, 588-599.

[21] Vora, H.H., Shukla, S.N., Brahambhatt, B.V., Mehta, S.H., Patel, N.A., Parikh, S.K., Shah, K.N. and Shah, P.M. (2010) Clinical Relevance of FLT3 Receptor Protein Expression in Indian Patients with Acute Leukemia. Asia-Pacific Journal of Clinical Oncology, 6, 306-319. https://doi.org/10.1111/j.1743-7563.2010.01322.x

[22] Peng, H.-L., Zhang, G.-S., Gong, F.-J., Shen, J.-K., Zhang, Y., Xu, Y.-X., Zheng, W.-L., Dai, C.-W., Pei, M.-F. and Yang, J.-J. (2008) Fms-Like Tyrosine Kinase (FLT) 3 and FLT3 Internal Tandem Duplication in Different Types of Adult Leukemia: Analysis of 147 Patients. Croatian Medical Journal, 49, 650-669. 
https://doi.org/10.3325/cmj.2008.5.650

[23] Terwilliger, T. and Abdul-Hay, M. (2017) Acute Lymphoblastic Leukemia: A Comprehensive Review and 2017 Update. Blood Cancer Journal, 7, e577.

https://doi.org/10.1038/bcj.2017.53

[24] Jaime-Pérez, J.C., Pinzón-Uresti, M.A., Jiménez-Castillo, R.A., Colunga-Pedraza, J.E., González-Llano, Ó. and Gómez-Almaguer, D. (2018) Relapse of Childhood Acute Lymphoblastic Leukemia and Outcomes at a Reference Center in Latin America: Organomegaly at Diagnosis is a Significant Clinical Predictor. Hematology, 23, 1-9. https://doi.org/10.1080/10245332.2017.1333294

[25] Fukuda, S., Broxmeyer, H.E. and Pelus, L.M. (2005) Flt3 Ligand and the Flt3 Receptor Regulate Hematopoietic Cell Migration by Modulating the SDF-1 $\alpha$ (CXCL12)/CXCR4 Axis. Blood, 105, 3117-3126. https://doi.org/10.1182/blood-2004-04-1440

[26] Dias, S., Hattori, K., Zhu, Z., Heissig, B., Choy, M., Lane, W., Wu, Y., Chadburn, A., Hyjek, E., Gill, M., Hicklin, D.J., Witte, L., Moore, M.A.S. and Rafii, S. (2000) Autocrine Stimulation of VEGFR-2 Activates Human Leukemic Cell Growth and Migration. Journal of Clinical Investigation, 106, 511-521. https://doi.org/10.1172/JCI8978

[27] Huang, Y., Chen, X., Dikov, M.M., Novitskiy, S.V., Mosse, C.A., Yang, L. and Carbone, D.P. (2007) Distinct Roles of VEGFR-1 and VEGFR-2 in the Aberrant Hematopoiesis Associated with Elevated Levels of VEGF. Blood, 110, 624-631. https://doi.org/10.1182/blood-2007-01-065714

[28] Fragoso, R., Pereira, T., Wu, Y., Zhu, Z., Cabeçadas, J. and Dias, S. (2006) VEGFR-1 (FLT-1) Activation Modulates Acute Lymphoblastic Leukemia Localization and Survival within the Bone Marrow, Determining the Onset of Extramedullary Disease. Blood, 107, 1608-1616. https://doi.org/10.1182/blood-2005-06-2530

[29] Tang, Y.-T., Jiang, F., Guo, L., Si, M.-Y. and Jiao, X.-Y. (2013) The Soluble VEGF Receptor 1 and 2 Expression in Cerebral Spinal Fluid as an Indicator for Leukemia Central Nervous System Metastasis. Journal of Neuro-Oncology, 112, 329-338. https://doi.org/10.1007/s11060-013-1066-x

[30] Dolence, J.J., Gwin, K., Frank, E. and Medina, K.L. (2011) Threshold Levels of Flt3-Ligand Are Required for the Generation and Survival of Lymphoid Progenitors and B-Cell Precursors. European Journal of Immunology, 41, 324-334. https://doi.org/10.1002/eji.201040710

[31] Takahashi, S. (2011) Downstream Molecular Pathways of FLT3 in the Pathogenesis of Acute Myeloid Leukemia: Biology and Therapeutic Implications. Journal of Hematology \& Oncology, 4, 13. https://doi.org/10.1186/1756-8722-4-13

[32] Scavelli, C., Vacca, A., Di Pietro, G., Dammacco, F. and Ribatti, D. (2004) Crosstalk between Angiogenesis and Lymphangiogenesis in Tumor Progression. Leukemia, 18, 1054-1058. https://doi.org/10.1038/sj.leu.2403355

[33] Siekmann, I.-K., Dierck, K., Prall, S., Klokow, M., Strauss, J., Buhs, S., Wrzeszcz, A., Bockmayr, M., Beck, F., Trochimiuk, M., Gottschling, K., Martens, V., Khosh-Naucke, M., Gerull, H., Müller, J., Behrmann, L., Blohm, M., Zahedi, R.P., Jeremias, I., Sickmann, A., Nollau, P. and Horstmann, M.A. (2018) Combined Inhibition of Receptor Tyrosine and p21-Activated Kinases as a Therapeutic Strategy in Childhood ALL. Blood Advances, 2, 2554-2567.

https://doi.org/10.1182/bloodadvances.2018020693

[34] Noronha, E.P., Andrade, F.G., Zampier, C., de Andrade, C.F.C.G., Terra-Granado, E., Pombo-de-Oliveira, M.S., Melaragno, R., Aranciba, A.M., de Oliveira, C.T., Ikoma, M.R.V., Nóbrega, A.G., Fialho, E.C.C., Neves, G.R., Magalhães, I.M.Q., 
Cordoba, J.C., de Brito, P.C., Dias, A.C.S., Costa, J.T., Souza, L.N.S., Santos, M. and Basegio, R.M. (2016) Immunophenotyping with CD135 and CD117 Predicts the FLT3, IL-7R and TLX3 Gene Mutations in Childhood T-Cell Acute Leukemia. Blood Cells, Molecules and Diseases, 57, 74-80.

https://doi.org/10.1016/j.bcmd.2015.12.003

[35] Zhang, J., Zhang, Y., Zhang, M., Liu, C., Liu, X., Yin, J., Wu, P., Chen, X., Yang, W., Zhang, L., Guo, Y., Zou, Y., Chen, Y., Cao, Y., Cheng, T. and Zhu, X. (2018) FLT3 Pathway Is a Potential Therapeutic Target for PRC2-Mutated T-Cell Acute Lymphoblastic Leukemia. Blood, 132, 2520-2524.

https://doi.org/10.1182/blood-2018-04-845628

[36] Blatt, K., Menzl, I., Eisenwort, G., Cerny-Reiterer, S., Herrmann, H., Herndlhofer, S., Stefanzl, G., Sadovnik, I., Berger, D., Keller, A., Hauswirth, A., Hoermann, G., Willmann, M., Rülicke, T., Sill, H., Sperr, W.R., Mannhalter, C., Melo, J.V., Jäger, U., Sexl, V. and Valent, P. (2018) Phenotyping and Target Expression Profiling of $\mathrm{CD} 4^{+} / \mathrm{CD} 38^{-}$and $\mathrm{CD} 34^{+} / \mathrm{CD} 38^{+}$Stem- and Progenitor Cells in Acute Lymphoblastic Leukemia. Neoplasia, 20, 632-642. https://doi.org/10.1016/j.neo.2018.04.004

[37] Wu, S., Geßner, R., Von Stackelberg, A., Kirchner, R., Henze, G. and Seeger, K. (2005) Cytokine/Cytokine Receptor Gene Expression in Childhood Acute Lymphoblastic Leukemia: Correlation of Expression and Clinical Outcome at First Disease Recurrence. Cancer, 103, 1054-1063. https://doi.org/10.1002/cncr.20869 


\section{Supplementary}

(A)

Blast \% expressing FLT3

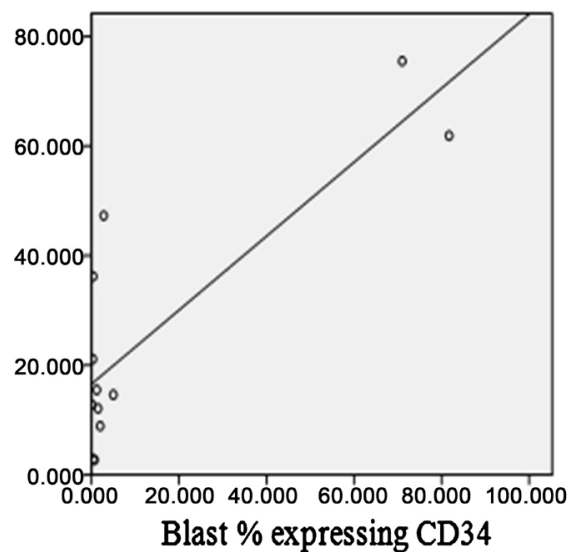

(B) Blast \% expressing VEGFR-1

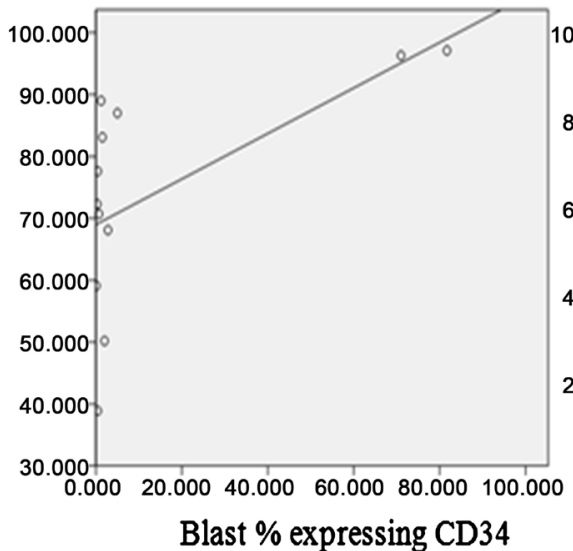

(C)

Blast \% expressing VEGFR-2

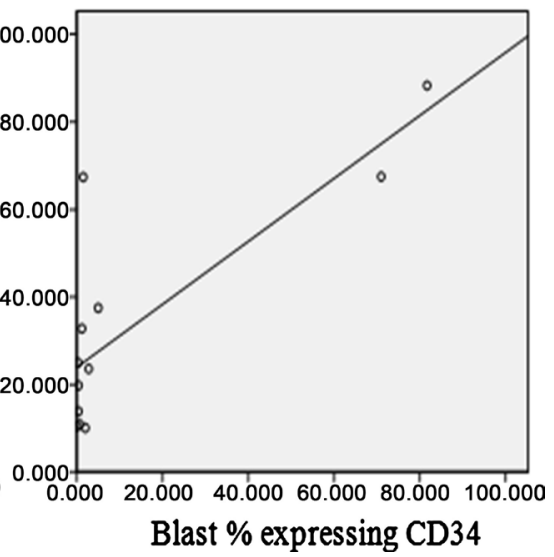

Figure S1. Correlation of blast \% expressing CD34 and blast \% expressing FLT3, VEGFR-1 and VEGFR-2 in T-ALL. (A) Positive correlation between blast \% expressing FLT3 and blast \% expressing CD34 in T-ALL $(r=0.826 ; \mathrm{p}=0.001)$. (B) Positive correlation between blast \% expressing VEGFR-1 and blast \% expressing CD34 in T-ALL $(r=0.596 ; \mathrm{p}=0.041)$. (C) Positive correlation between blast \% expressing VEGFR-2 and blast \% expressing CD34 in T-ALL $(r=0.798 ; p=0.002)$. 